\title{
Gain-of-function miRNA signature by mutant p53 associates with poor cancer outcome
}

\author{
Yao Zhang ${ }^{1}$, Ye Hu ${ }^{1,2}$, Jing-Yuan Fang ${ }^{1}$, Jie $\mathbf{X u}^{1}$ \\ ${ }^{1}$ State Key Laboratory for Oncogenes and Related Genes, Key Laboratory of Gastroenterology and Hepatology, Ministry of \\ Health, Division of Gastroenterology and Hepatology, Renji Hospital, School of Medicine, Shanghai Jiao Tong University, \\ Shanghai Institute of Digestive Disease, Shanghai Cancer Institute, Shanghai, China \\ ${ }^{2}$ Xinhua Hospital, Shanghai Jiao Tong University School of Medicine, Shanghai 200092, China
}

Correspondence to: Jie Xu, e-mail: xujieletter@gmail.com

Keywords: cancer prognosis, miRNA, mutation, non-negative matrix factorization, p53

Received: October 08, 2015

Accepted: January 15, 2016

Published: January 31, 2016

\section{ABSTRACT}

Missense mutation of p53 not only impairs its tumor suppression function, but also causes oncogenic gain of function (GOF). The molecular underpinning of mutant p53 (mutp53) GOF is not fully understood, especially for the potential roles of non-coding genes. Here we identify the microRNA expression profile (microRNAome) of mutp53 on Arg282 by controlled microarray experiments, and clarify the prognostic significance of mutp53-regulated miRNAs in cancers. A predominant repression effect on miRNA expression was found for mutant p53, with 183 significantly downregulated and only 12 upregulated miRNAs. Mutp53 and wild-type (wtp53) commonly upregulate let-7i, and other two miRNAs were upregulated by wtp53 but repressed by mutp53 (miR-610 and miR-3065-3p). Based the mutp53-regulated miRNA signature, a non-negative matrix factorization (NMF) model classified gastric cancer (GC) cases into subgroups with significantly different Disease-free survival (Kaplan-Meier test, $P=0.013)$. In contrast, the NMF model based on all miRNAs did not associate with cancer outcome. The mutp53 miRNA signature associated with the outcomes of breast cancer $(P=0.024)$ and hepatocellular cancer $(P=0.012)$. The miRPath analysis revealed that mutp53-suppressed miRNAs associate with Hippo, TGF- $\beta$ and stem cell signaling pathways. Taken together, our results highlight a miRNA-mediated GOF mechanism of mutant p53 on Arg282, and suggest the prognostic potential of mutp53-associated miRNA signature.

\section{INTRODUCTION}

The TP53 gene that encodes the p53 tumor suppressor protein is the most commonly mutated gene in all human cancers [1], and missense mutation causing substitution of single amino acid represents the major type of mutations of TP53 gene [2]. The wild-type p53 is a master regulator of human genomic integrity [3], which is stabilized an accumulated in the nucleus in response to genomic stress or oncogenic signaling [4]. The p53 protein was firstly recognized as a transcription factor, which binds to DNA in a sequence-specific manner in its tetrameric form. The regulation of p53 monomertetramer assembly is regulated by c-abl and RhoGAPs [5]. The downstream target genes of p53 are involved in multiple pathways such as cell cycle arrest, apoptosis, and metabolism [6]. The cytoplasmic function of $\mathrm{p} 53$ protein associates with mitochondria outer membrane, where it binds BCL-XL and induces cytochrome $\mathrm{C}$ release and initiates apoptosis [7].

Missense mutation of p53 not only causes loss of tumor suppression function (LOF), but also causes gain of oncogenic function (GOF) [8]. Evidence based on both transgenic mouse model and human cancer data consistently support the GOF effect of mutant p53 (mutp53). When compared to p53-null mice [9], mice harboring the hotspot mutant p53 displayed more spontaneous tumors and shorter survival. In addition, different p53 mutants have been confirmed to associate with different outcomes [10]. In a previous study, we have demonstrated that the hotspot p53 mutation on Arg282 (R282) associated with significantly earlier cancer onset in Li-Fraumeni families 
that carry germline p53 mutations [11]. Moreover, the R282 mutation also associated with shorter survival of cancer patients, as reported in our previous study. Due to the variable signaling pathways and clinicopathological features of different $\mathrm{p} 53$ mutations, it has been proposed that p53 mutations should be considered as different oncogenes and biomarkers in cancers [12]. In a structural perspective, the R282 mutation may destabilize of the protein structure [13], induce protein aggregation [14], and affect its binding to BCL-XL in mitochondria [15]. However, the signaling pathways associated with R282 mutant are largely unknown, presenting a major challenge for developing targeted therapy.

MicroRNAs (miRNAs) are a class of non-coding RNAs with 20-25 nucleotides in length that function in RNA silencing and post-transcriptional regulation of gene expression [16] The miRNA expression profile (miRNAome) is significantly altered in cancers [17-19], and it is known that miRNAs may play causative roles in cancers by regulating multiple signaling pathways $[20,21]$. The competing endogenous RNA (ceRNA) regulatory networks involving protein-coding messenger RNAs, long-noncoding RNAs and miRNAs are emerging factors that may contribute to cancer development $[22,23]$. The miRNAome may be affected by multiple factors in cancer, including gene copy alteration $[24,25]$, transcription factor dysregulation, and the dynamics of ceRNA network. Besides, microRNAs have been generally accepted to be involved in the p53-regulated network, like contributing to the down-regulations of mRNA and protein expression observed after p53 activation [26]. Otherwise, we found previously that mutp53 R248W could transactivate the GAPLINC long non-coding RNA and promote the expression of CD44 oncogene [27]. Therefore, mutp53 GOF may not only involve protein-coding genes (PCGs) but also non-coding genes. Although some studies have already explored the correlations between cancers and miRNA, signature [28] and survival predictions [29], it is unknown to which extent mutp53 may affect the expression of miRNAs, and whether the mutp53 related miRNA signature may be prognostic in cancers.

In the present study, we characterize the mutp53 Arg282-regulated miRNAome in cancer cells, and analyze the prognostic significance of mutp53-regulated miRNA signature. The signaling pathways associated with the miRNA signature were analyzed with the miRPath algorithm, based on the enrichment of miRNA-mRNA target pairs in KEGG pathway. Through these approaches, we aim to identify the miRNA-mediated GOF mechanisms of $\operatorname{Arg} 282$ hotspot p53 mutation.

\section{RESULTS}

\section{Identification of mutp53 $\mathrm{R} 282 \mathrm{~W}$ regulated miRNAs}

To identify mutp53 R282W regulated miRNAs in an unbiased manner, we performed stable transfection of the p53-null H1299 cells with an expression vector encoding the p53 R282W mutant. After total RNA isolation, microarray assay (Affymetrix miRNA 4.0) was used to measure the expression profile of miRNAs. The cells transfected with empty vector was used as control, and each experimental group had triple biological repeats (schematics of experimental procedures shown in Figure 1A). Because foldchange ranking combined with a non-stringent statistical $P$-value [30-32] has been found to be more reliable for microarray-based differential expression analysis, we used the widely-accepted criteria of fold-change $>2$ (or $<0.5$ ) and $P$-value $<0.05$ [33-36]. Interestingly, we found that mutant p53 R282W had a predominant repression effect on miRNA epxression, with 183 downregulated miRNAs (Supplementary Tables S1) but only 12 upregulated miRNAs (Supplementary Tables S2). We have already uploaded the microRNA data to the GEO website (Series GSE 73876).

\section{Most mutp53-regulated miRNAs are unrelated to wtp53}

We further analyzed if mutp53 R282W-regulated miRNAs might partially overlap with those regulated by the wild-type p53 (wtp53). The wtp53-regulated miRNAs were determined based on the raw data of a recently published paper, using the same criteria as mutp53 [37]. Interestingly, only one miRNA, namely hsa-let-7i was found commonly upregulated by wtp53 and mutp 53 . Two other miRNAs (hsa-miR-610 and hsa-miR-3p) were downregulated by mutp53, but upregulated by wtp53 . No miRNA was found commonly downregulated by mutp53 and wtp53 (Figure 1B). Of note, the mutp53 regulated miRNAs were found with uneven chromosomal distribution. While chromosome 1 contained 21 miRNAs that were regulated by mutp53, chromosomes 21 and 22 only contained one such miRNA, respectively (Figure 1C, listed in Supplementary Table S3). The vast majority of mutp53 regulatory targets were unrelated to wtp53, suggesting that mutp53 may acquire GOF effects through regulating miRNAs.

\section{Mutp53-regulated miRNA signature associates with prognosis of gastric cancer}

We further questioned whether the mutp53regulated miRNAs may associate with the prognosis of cancers. The expression profiles of miRNAs were obtained from the gastric adenocarcinoma dataset of the cancer genome atlas (TCGA) that included 470 cancer cases [38]. Among the 195 mutp53-regulated miRNAs, 38 were found abundantly expressed in the gastric cancer samples (list in Supplementary Table S4). This partial mutp53 signature containing 38 miRNAs was then used to calculate the similarity between cancer samples (schematic diagram in Figure 2A). Since the non-negative matrix factorization (NMF) model has been successfully applied to classify cancer samples with clinical significance [39], we also employed the NMF method in the present study 
(detailed procedures described in the Methods section). Interestingly, the partial mutp53 miRNA signature classified the cancer samples into 3 or 4 subgroups with significantly different disease-free survival $(P=0.015$ and $P=0.013$, respectively). In contrast, NMF clustering revealed subgroups with no association with cancer outcome (Figure 3).

Of note, the miRNA signature-based classification displayed stronger association with cancer outcome than the traditional AJCC staging (Figure 3) and microsatellite instability (Supplementary Figure S1). When the p53 gene status was categorized as wild type, missense mutation or truncating mutation, patients with different p53 status displayed no significant difference in DFS. Likewise, the p53 gene copy number, mRNA expression, and protein expression displayed no significant association with DFS (Supplementary Figure S1). These may be due to the complexity of p53 regulation at genetic, epigenetic, and protein levels. Thus, one such factor alone may not perfectly mark its functional status.

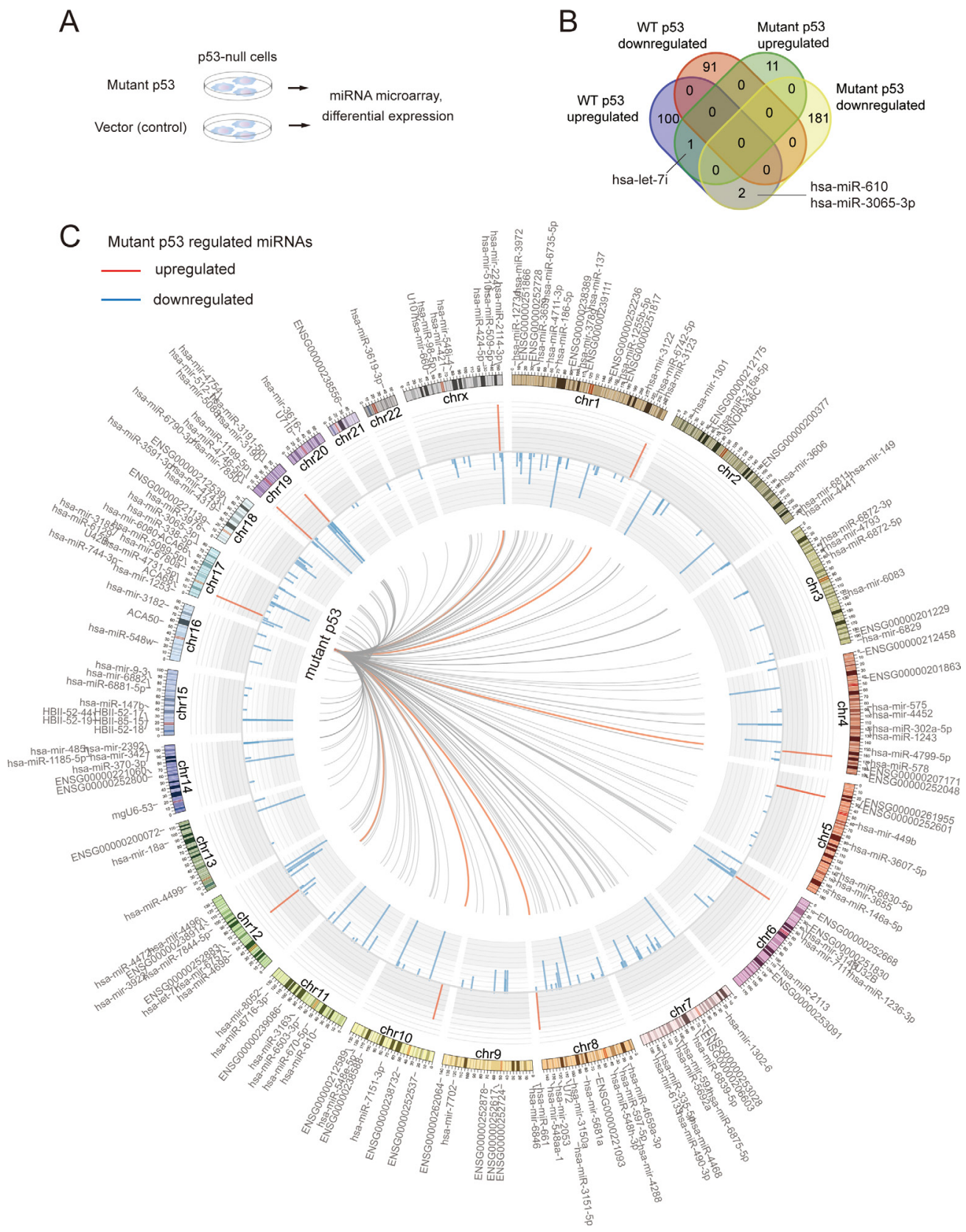

Figure 1: The miRNAome of mutp53 largely differs from that of wtp53. (A) Schematic representation of the study procedures. Mutant p53 or empty vector was stably transfected to p53-null H1290 cancer cells, followed by Affymetrix miRNA 4.0 microarray study and differential expression analysis. (B) Venn's diagram showing the common and unique miRNAs regulated by either mutp53 or wtp53. (C) The Circos map indicates the miRNAs that were upregulated (red) or downregulated (blue) by mutp53 R282W. The chromosomal locations of regulated miRNAs are also indicated. 
A

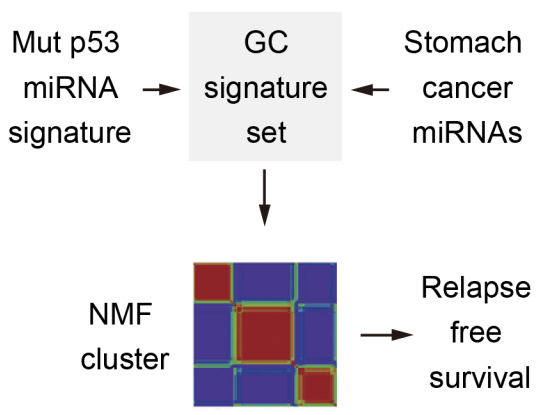

C Mutant p53 miRNA signature, 3 classes

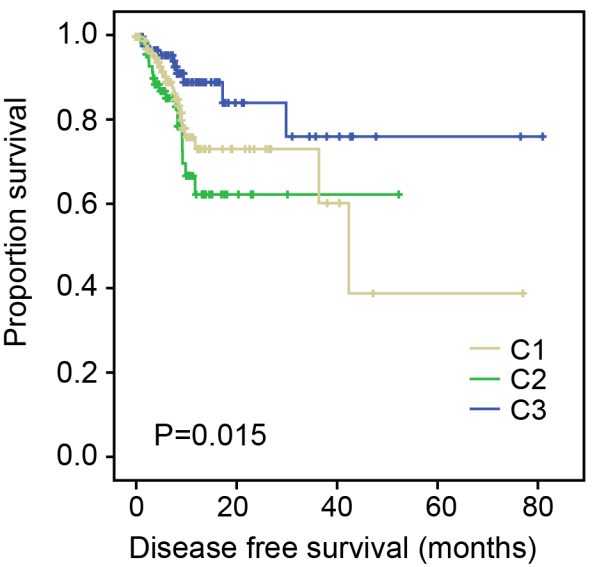

E Mutant p53 miRNA signature, 4 classes

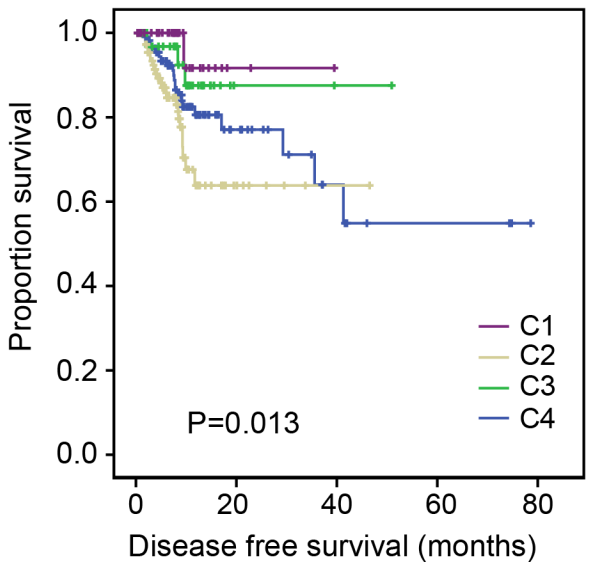

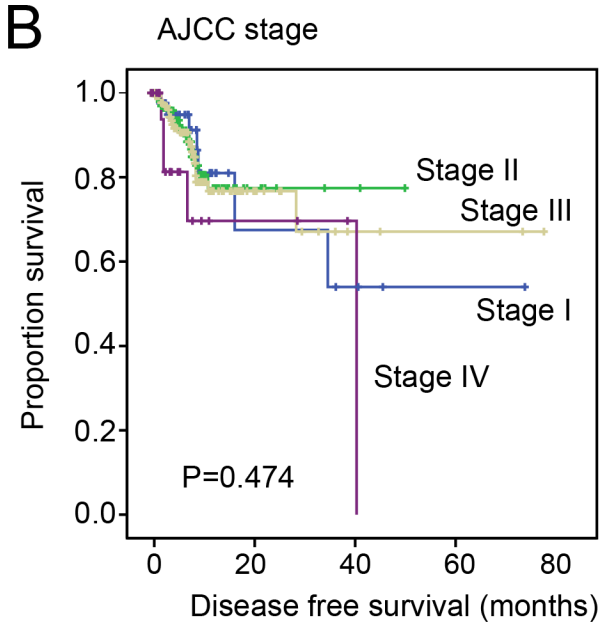

D All miRNAs, 3 classes

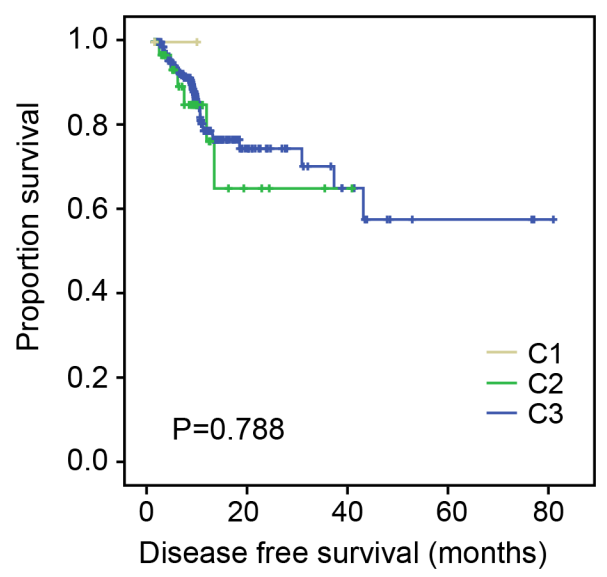

$\mathrm{F}$

All miRNAs, 4 classes

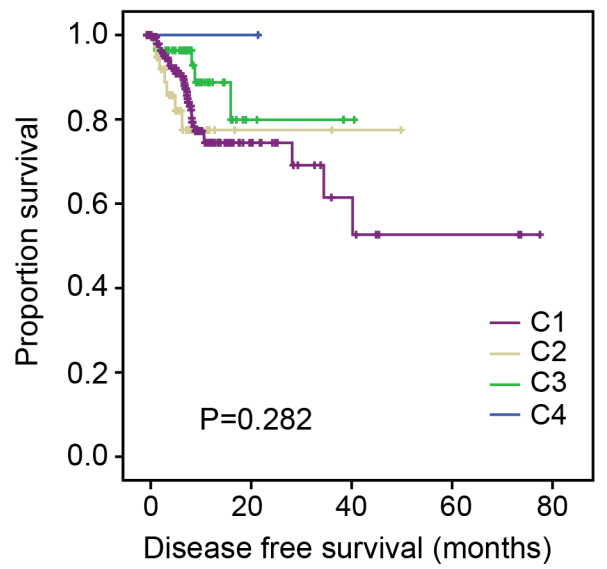

Figure 2: The miRNA signature of mutp53 associated with disease-free survival of gastric cancer patients. Comparison of survival in all panels were performed using Kaplan-Meier test. (A) The schematic diagram showing the analysis flow. A partial mutp53 signature was derived by overlapping mutp53-regulated miRNAs and abundantly expressed miRNAs in gastric cancer. a non-negative matrix factorization (NMF) model was used to classify the cancer patients based on the miRNA signature, followed by DFS comparison between different groups. (B) The survival of gastric cancer patients with different AJCC stages were compared using Kaplan-Meier survival test ( $P$-value indicated). (C-D) DFS of patients in 3 subgroups determined by NMF clustering based on either the miRNA signature (C) or all miRNAs (D). (E-F) Comparison of patient DFS in 4 subgroups as determined based on miRNA signature (E) or all miRNAs (F). 
A Breast adenocarcinoma (90 cases)

Mutant p53 signature (45 miRNAs)

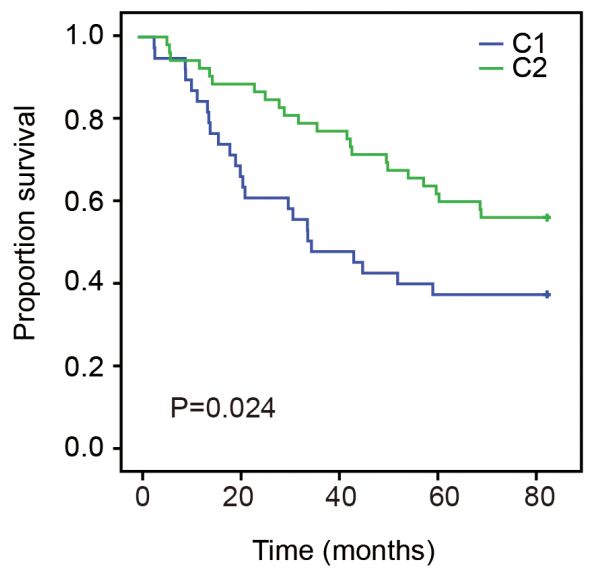

C Hepatocellular carcinoma
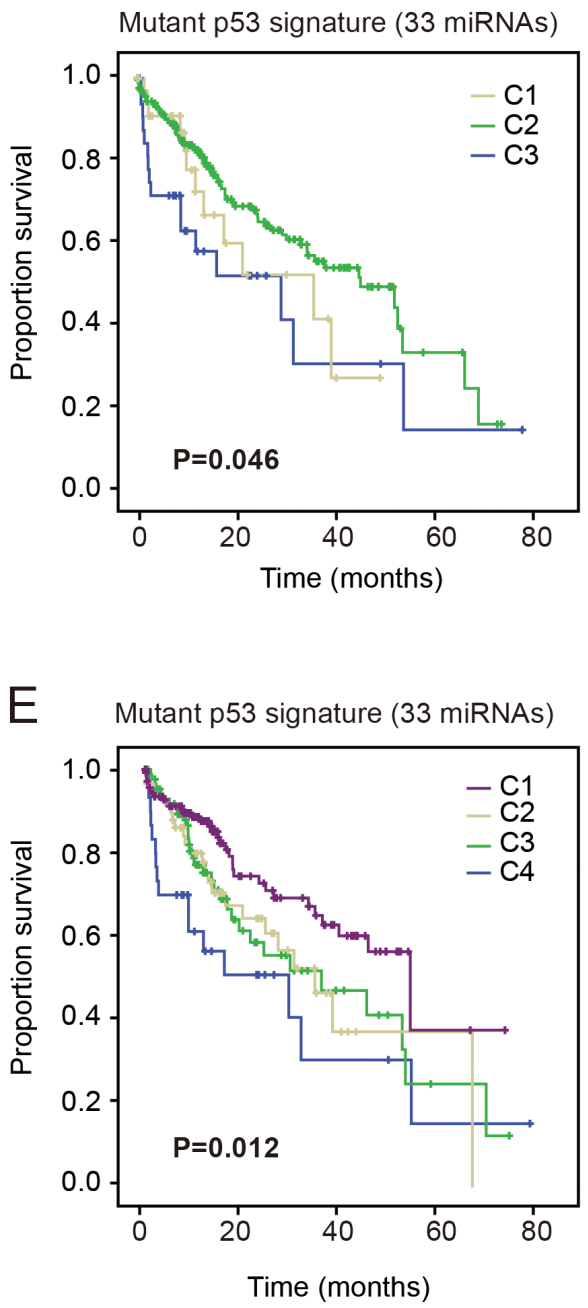

B
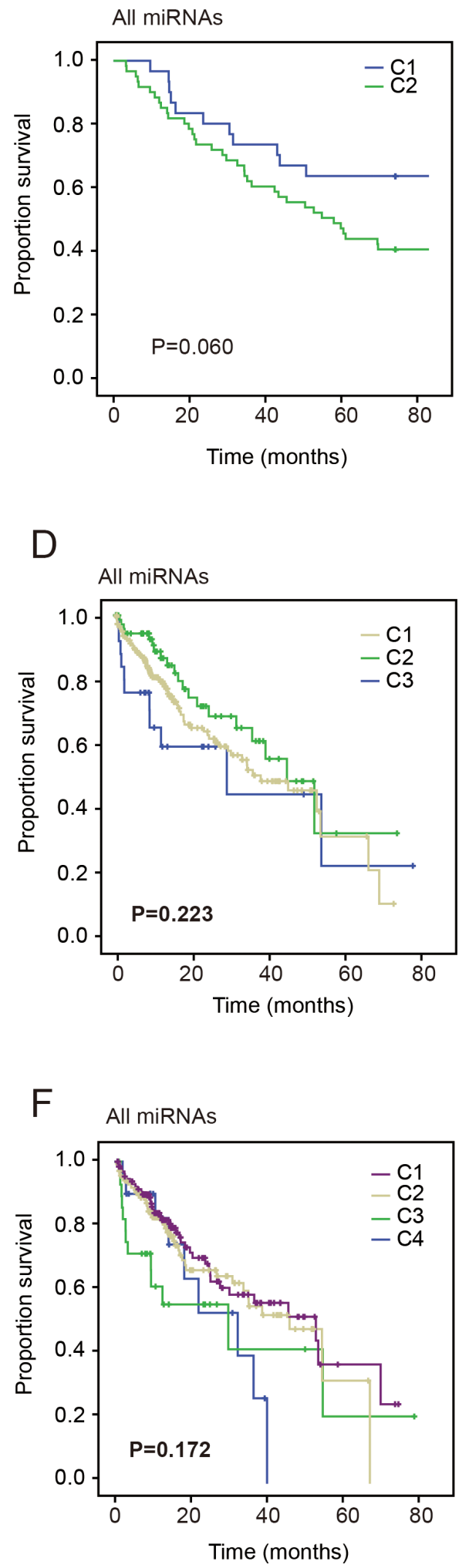

Figure 3: The mutp53 miRNA signature associates with prognosis of breast and liver cancers. Comparison of survival in all panels were performed using Kaplan-Meier test. (A-B) Disease-free survival of breast cancers with lymphnode involvement. The subgroups determined by miRNA signature displayed significantly different DFS (A), but the subgroups determined by all miRNAs did not significantly associate with DFS (B). (C-D) Survival of patients in 3 subgroups of liver cancer patients classified by the mutp53 miRNA signature (C) or all miRNAs (D). (E-F) The comparison of patient survival in 4 subgroups determined based on miRNA signature (E) or all miRNAs (F). 


\section{Mutp53 miRNA signature in the prognosis of breast and liver cancers}

Since p53 is also frequently mutated in multiple cancer types, we also examined the association of mutp53 miRNA signature with the prognosis of other cancers. The breast cancer dataset reported by Buffa and colleagues [40] contained both miRNA expression profile and patient DFS, and thus was included in this study. The NMF model divided the patients into 2 subgroups, because more groups caused significant decrease in cophenetic correlation. As a result, the partial mutp53 signature (listed in Supplementary Table S4) classified breast cancer patients with significantly different DFS $(P=0.024$, Kaplan-Meier survival test). In contrast, the classification model based on all miRNAs showed no significant association with DFS $(P=0.06$, Figure $3 \mathrm{~A}$ and $3 \mathrm{~B})$.

Further, we analyzed the prognostic effect of mutp53 miRNA signature in liver cancer. Since the disease-free survival data of the TCGA liver cancer patients were more incomplete (247 of 377 cases unavailable), we analyzed the overall survival of patients. Again, the partial mutp53 signature (miRNAs listed in Supplementary Table S4) divided patients into 3 or 4 groups with significantly different survival $(P=0.046$ and $P=0.012$ ), but the model based on all miRNAs displayed no significant association (Figure $3 \mathrm{C}-3 \mathrm{~F}$ ). These results consistently suggest that mutp 53 miRNA signature is associated with cancer outcome.

\section{Mutp53-repressed miRNAs target multiple cancer-related pathways}

To probe the signaling pathways of mutp53regulated miRNAs, we performed pathway enrichment analysis for miRNAs using the miRPath algorithm, which is based on more than 600,000 experimentally supported miRNA targets from DIANA-TarBase [41]. Since the mutp53 displayed predominant suppression effect on miRNAs, we firstly analyzed the pathways that were targeted by the downregulated miRNAs (in positive association with mutp53 function). The hippo signaling pathway was the most significantly associated gene set, containing 11 genes that were targeted by 96 miRNAs (Figure 4A). These miRNAs represent half of all mutp53-repressed miRNAs, indicating a strong association between mutp53 and the Hippo signaling pathway. The pathways belonging to TGF- $\beta$ signaling, regulation of pluripotency of stem cells, proteoglycans in cancer were also significantly associated with mutp53repressed miRNAs (Figure 4A). The miRNAs upregulated by mutp53 were associated with specific metabolic pathways (steroid hormone, mycin type O-glycan, and glycosphingolipid biosynthesis), as well as the ErbB signaling (Figure 4B). Note that such associations were less significant, due to the smaller number of miRNAs upregulated by mutp53. In fact, the hippo signaling pathway has been reported to regulate the pluripotency of stem cells [42] and to interplay with the TGF- $\beta$ pathway [43, 44], thus the pathways associated with mutp53-repressed miRNAs might reflect the significant involvement of hippo signaling.

\section{DISCUSSION}

The mechanisms of mutp53 gain-of-function effect are important for developing targeted therapies against advanced cancers, and our study presents the first unbiased characterization of mutp53 R282W-regulated miRNAs. We demonstrate that a mutp53 miRNA signature can identify cancer subgroups with significantly different outcomes, and the hippo signaling is associated with the mutp53 signature.

Firstly, our data revealed a novel miRNAome of mutp53 that substantially differs from that of wtp53. Among the 195 mutp53-regulated miRNAs, only 3 were related to wtp53. As reported previously, the wtp53 regulates a broad panel of miRNAs with potential tumor suppressing roles, including miR-34a-5p, miR-182-5p, miR-203a, miR-222-3p, and miR-432-5p, etc [45-47]. Our data suggest that on a p53-null background, ectopic expression of mutp53 repressed the expression of many miRNAs that are not regulated by wtp53. This may be explained by the two major GOF models: 1) mutp53 may bind new gene promoters and acquire a novel transcriptome; 2) mutp53 may interact with other proteins and indirectly regulate gene expression through its mutant interactome $[12,48]$. Since miRNAs belong to competing endogenous RNA (ceRNA) networks, the intermediate pathways between mutp3 and miRNA expression might be complicated. It deserves further investigation whether other coding genes or long noncoding genes might be involved in the effects of mutp53 on miRNA expression.

Moreover, our results demonstrate that a mutp53 GOF signature composed of miRNAs could successfully divide patients into subgroups with clinical relevance. It has been suggested that GOF mutation of p53 associates with worse cancer outcomes, but our results revealed for the first time that mutp53-regulated miRNAs have prognostic significance. Indeed, the miRNA signature displayed stronger association with gastric cancer DFS, over p53 genetic status or its expression level. The expression of miRNAs were strongly influenced by mutp53, and thus a miRNA signature could reflect the functional status of $\mathrm{p} 53$. Other factors such as p53 genetic status, copy number alteration, mRNA level, or protein level were not ideal markers for p53 function, which is actually a result of interaction between the above-mentioned factors. It should be noted that miRNA expression profiles differ substantially between cancer types, thus the miRNA signatures for gastric, liver and breast cancers were distinct subsets of the mutp53regulated miRNAs. Even though, the partial mutp53 signature displayed prognostic significance in these cancer types. 
Signaling pathways of downregulated miRNAs

\begin{tabular}{lccc}
\hline KEGG pathway & p-value & \#miRNAs & \#genes \\
\hline Hippo signaling pathway & $0.00 \mathrm{E}+00$ & 96 & 11 \\
TGF-beta signaling pathway & $1.11 \mathrm{E}-16$ & 54 & 9 \\
Regulating pluripotency of stem cells & $5.55 \mathrm{E}-16$ & 86 & 10 \\
Proteoglycans in cancer & $5.18 \mathrm{E}-14$ & 127 & 9 \\
Mucin type O-Glycan biosynthesis & $1.95 \mathrm{E}-08$ & 19 & 10 \\
Morphine addiction & $1.02 \mathrm{E}-07$ & 46 & 10 \\
ECM-receptor interaction & $6.51 \mathrm{E}-07$ & 34 & 5 \\
Lysine degradation & $2.16 \mathrm{E}-05$ & 20 & 7 \\
Glioma & $2.02 \mathrm{E}-03$ & 41 & 8 \\
Wnt signaling pathway & $3.65 \mathrm{E}-03$ & 75 & 5 \\
\hline
\end{tabular}

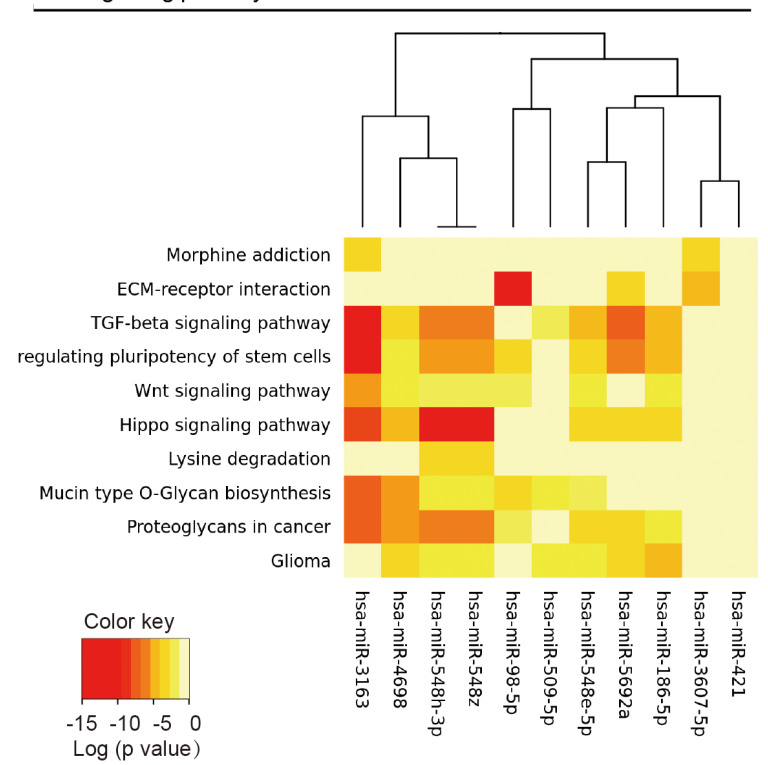

B Signaling pathways of upregulated miRNAs

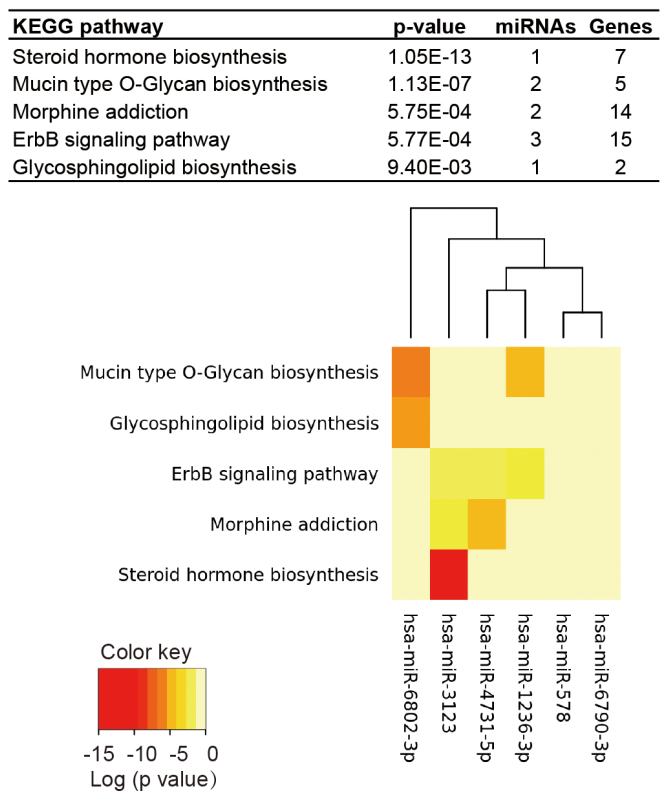

Figure 4: The signaling pathways associated with mutp53-regulated miRNAs. (A) KEGG signaling pathways that significantly associated with miRNAs repressed by mutp53. The results were based on miRPath, an experimentally supported tool for identifying miRNA-targeted pathways. The significance, number of targeted genes and miRNAs are shown in the upper panel, and the heat map in the lower panel indicate the involvement of top-ranked miRNAs in the indicated signaling pathway. (B) The pathway enrichment analysis of mutp53-upregulated miRNAs. The significance of each pathway and the association of top-ranked miRNAs are respectively shown in the upper and lower panels. 
Finally, our pathway analysis revealed that mutp53 $\mathrm{R} 282 \mathrm{~W}$ associated with the hippo signaling, which is involved in regulating pluripotency of stem cells and cancer aggressiveness $[49,50]$. The experimentally supported miRPath algorithm identified multiple genes in the hippo pathway that could be targeted by miRNAs in the mutp53 signature, suggesting that mutp $53 \mathrm{R} 282 \mathrm{~W}$ may acquire GOF effect through this oncogenic route. This finding also raises the possibility of targeting hippo signaling pathway in cancer cases bearing the p53 R282 hot-spot mutation.

In conclusion, the hot-spot mutp53 R282W regulates a novel miRNAome that largely differs from that of wtp53, and this mutant GOF signature associates with poor prognosis of multiple cancer types. In future research, the mechanisms underlying this mutant-specific miRNAome should be investigated, which may facilitate the development of more effective targeted cancer therapies.

\section{METHODS}

\section{Plasmid construction}

The pcDNA3-HA-p53 expression vector was constructed by inserting PCR-amplified p53 cDNA sequence into pcDNA3 vector (Invitrogen, Carlsbad, CA, USA). The p53 R282W mutant was derived by sitedirected mutagenesis PCR reaction using platinum PWO SuperYield DNA polymerase (Roche, Basel, Switzerland) according to the product manual. The plasmid was sequenced to confirm if the designed mutation is present, without any other unwanted mutation.

\section{Cell culture and transfection}

The human H1299 (p53-null) cells were maintained in DMEM medium (Gibco, Gaithersburg, MD, USA) supplemented with $10 \%$ fetal bovine serum (Invitrogen) and cultured in a humidified incubator at $37^{\circ} \mathrm{C}$ under $5 \% \mathrm{CO} 2$. Before transfection, cells were seeded into normal growth medium at $50 \%$ confluence in six-well tissue plates. The FuGENE HD transfection reagent (Promega, Fitchburg, WI, USA) was applied according to the product manual. Briefely, the transfection complex was made by $1 \mu \mathrm{g}$ plasmid, $3 \mu \mathrm{l} \mathrm{FuGENE} \mathrm{HD} \mathrm{and} 100 \mu \mathrm{l}$ media. Six hours after the complex was added to the cells, normal culture media was used to culture cells for additional $48 \mathrm{~h}$, followed by gene expression analysis. For stable transfection, the $600 \mu \mathrm{g} / \mathrm{mL}$ G418 was used to culture cells for four weeks, and the expression of mutp53 in stable strains was confirmed by quantitative PCR.

\section{MicroRNA-microarray experiment}

The H1299 cells stably transfected with mutp53 $\mathrm{R} 282 \mathrm{~W}$ or the control vector were respectively analyzed by Affymetrix GeneChip miRNA 4.0 microarray with three biological replications. A total of $200 \mathrm{ng}$ small RNA was used in sample preparation with a FlashTag Biotin RNA Labeling Kit for Affymetrix GeneChip miRNA arrays (Genisphere). The labeled RNA was consequently hybridized for sixteen hours to an Affymetrix GeneChip miRNA array according to the product manual. Microarrays were washed and stained in the Affymetrix Fluidics Station 450, and scanned on the Affymetrix G3000 GeneArray Scanner. The image files were analyzed using the Affymetrix software (Expression Console), Robust Multi-array Average (RMA) background correction, log-2 transformations and global normalization methods were performed for data pre-processing, and normalization.

\section{Unsupervised clustering by non-negative matrix factorization (NMF) algorithm}

NMF is a matrix factorization algorithm that focuses on the analysis of data matrices whose elements are nonnegative, and the principles and the detailed algorithm has been described previously [51]. We employed the NMF algorithm module of the MEV 4.9 program package [52] to perform unsupervised clustering of cancer samples, with «divergence» as the update rule and maximum iteration of 1,000 . The optimal number of classes was determined according to the cophenetic correlation value, as described in our previous study [39].

\section{Survival analysis}

The survival analysis was carried out using the SPSS software package. In the Kaplan-Meier (log rank) survival test model, the censored status was indicated when the patient was still alive (or cancer-free) at the time of follow-up. The criteria of $P<0.05$ was used for judging statistical significance.

\section{Pathway analysis of miRNAs}

The signaling pathways in association with miRNAs were analyzed with the miRPath v3.0 web server as described previously [41]. Briefly, the down-regulated (or up-regulated) miRNAs in the mutp53 signature were used as input, and the microT-CDS method was used for determining target genes. The species was defined as "human", and the gene filter was set as "determine genes". The enrichment of target genes in different KEGG molecular pathways were ranked by the respective $P$-values (adjusted by false discovery rate, FDR). The criteria of $P<0.01$ (adjusted) was used for judging statistically significance.

\section{ACKNOWLEDGMENTS AND FUNDING}

This project was supported by grants from National Natural Science Foundation of China (81421001, $31371420,81320108024,81000861,81322036$, and 
81272383), Top-Notch Young Talents Program of China (ZTZ2015-48), Shanghai Municipal Education Commission-Gaofeng Clinical Medicine Grant Support (20152514), National Key Technology Support Program (2015BAI13B07) and Shanghai "Oriental Scholars" project $(2013 \mathrm{XJ})$.

\section{Authors' contributions}

YZ, and YH performed experiments; YZ, JYF and JX analyzed data. YZ and JX wrote the manuscript. JX conceived and supervised the study.

\section{CONFLICTS OF INTEREST}

The authors declare no competing financial interests.

\section{REFERENCES}

1. Grawenda AM, Moller EK, Lam S, Repapi E, Teunisse AF, Alnaes GI, Borresen-Dale AL, Kristensen VN, Goding CR, Jochemsen AG, Edvardsen H, Bond GL. Interaction between p53 mutation and a somatic HDMX biomarker better defines metastatic potential in breast cancer. Cancer research. 2015; 75:698-708.

2. Lu X, Liu DP, Xu Y. The gain of function of p53 cancer mutant in promoting mammary tumorigenesis. Oncogene. 2013; 32:2900-2906.

3. Blagosklonny MV. P53: an ubiquitous target of anticancer drugs. International journal of cancer. 2002; 98:161-166.

4. Park BJ, Oh YS, Park SY, Choi SJ, Rudolph C, Schlegelberger B, Kim S. AIMP3 haploinsufficiency disrupts oncogene-induced p53 activation and genomic stability. Cancer research. 2006; 66:6913-6918.

5. Xu J, Zhou X, Wang J, Li Z, Kong X, Qian J, Hu Y, Fang JY. RhoGAPs attenuate cell proliferation by direct interaction with $\mathrm{p} 53$ tetramerization domain. Cell reports. 2013; 3:1526-1538.

6. Bensaad K, Tsuruta A, Selak MA, Vidal MN, Nakano K, Bartrons R, Gottlieb E, Vousden KH. TIGAR, a p53inducible regulator of glycolysis and apoptosis. Cell. 2006; 126:107-120.

7. Pu J, Yuan A, Shan P, Gao E, Wang X, Wang Y, Lau WB, Koch W, Ma XL, He B. Cardiomyocyte-expressed farnesoid-X-receptor is a novel apoptosis mediator and contributes to myocardial ischaemia/reperfusion injury. Eur Heart J. 2013; 34:1834-1845.

8. Blagosklonny MV. p53 from complexity to simplicity: mutant p53 stabilization, gain-of-function, and dominantnegative effect. The FASEB Journal. 2000; 14:1901-1907.

9. Lang GA, Iwakuma T, Suh YA, Liu G, Rao VA, Parant JM, Valentin-Vega YA, Terzian T, Caldwell LC, Strong LC, ElNaggar AK, Lozano G. Gain of function of a p53 hot spot mutation in a mouse model of Li-Fraumeni syndrome. Cell. 2004; 119:861-872.
10. Demidenko ZN, Fojo T, Blagosklonny MV. Complementation of two mutant p53: Implications for loss of heterozygosity in cancer. FEBS letters. 2005; 579: 2231-2235.

11. Xu J, Qian J, Hu Y, Wang J, Zhou X, Chen H, Fang JY. Heterogeneity of Li-Fraumeni syndrome links to unequal gain-of-function effects of p53 mutations. Scientific reports. 2014; 4:4223.

12. Freed-Pastor WA, Prives C. Mutant p53: one name, many proteins. Genes \& development. 2012; 26:1268-1286.

13. Calhoun S, Daggett V. Structural effects of the L145Q, V157F, and R282W cancer-associated mutations in the p53 DNA-binding core domain. Biochemistry. 2011; 50: 5345-5353.

14. Xu J, Reumers J, Couceiro JR, De Smet F, Gallardo R, Rudyak S, Cornelis A, Rozenski J, Zwolinska A, Marine JC, Lambrechts D, Suh YA, Rousseau F, et al. Gain of function of mutant p53 by coaggregation with multiple tumor suppressors. Nature chemical biology. 2011; 7:285-295.

15. Xu J, Wang J, Hu Y, Qian J, Xu B, Chen H, Zou W, Fang JY. Unequal prognostic potentials of p53 gain-offunction mutations in human cancers associate with drugmetabolizing activity. Cell death \& disease. 2014; 5:e1108.

16. Wang JL, Hu Y, Kong X, Wang ZH, Chen HY, Xu J, Fang JY. Candidate microRNA biomarkers in human gastric cancer: a systematic review and validation study. PloS one. 2013; 8:e73683. doi: 10.18632/oncotarget.3005.

17. Murray BS, Choe SE, Woods M, Ryan TE, Liu W. An in silico analysis of microRNAs: mining the miRNAome. Molecular bioSystems. 2010; 6:1853-1862.

18. Bruce JP, Hui ABY, Shi W, Perez-Ordonez B, Weinreb I, Xu W, Haibe-Kains B, Waggott DM, Boutros PC, O'Sullivan B, Waldron J, Huang SH, Chen EX, et al. Identification of a microRNA signature associated with risk of distant metastasis in nasopharyngeal carcinoma. Oncotarget. 2015; 6:4537-4550. doi: 10.18632/oncotarget.3005.

19. Liu N, Boohaker RJ, Jiang C, Boohaker JR, Xu B. A radiosensitivity MiRNA signature validated by the TCGA database for head and neck squamous cell carcinomas. Oncotarget. 2015; 6:34649-34657. doi: 10.18632/ oncotarget.5299.

20. Hall DP, Cost NG, Hegde S, Kellner E, Mikhaylova O, Stratton Y, Ehmer B, Abplanalp WA, Pandey R, Biesiada J, Harteneck C, Plas DR, Meller J, et al. TRPM3 and miR204 establish a regulatory circuit that controls oncogenic autophagy in clear cell renal cell carcinoma. Cancer cell. 2014; 26:738-753.

21. Valeri N, Braconi C, Gasparini P, Murgia C, Lampis A, Paulus-Hock V, Hart JR, Ueno L, Grivennikov SI, Lovat F, Paone A, Cascione L, Sumani KM, et al. MicroRNA-135b promotes cancer progression by acting as a downstream effector of oncogenic pathways in colon cancer. Cancer cell. 2014; 25:469-483. 
22. Hu Y, Tian $\mathrm{H}, \mathrm{Xu}$ J, Fang JY. Roles of competing endogenous RNAs in gastric cancer. Briefings in functional genomics. 2015.

23. Xia T, Liao Q, Jiang X, Shao Y, Xiao B, Xi Y, Guo J. Long noncoding RNA associated-competing endogenous RNAs in gastric cancer. Scientific reports. 2014; 4:6088.

24. Liang L, Fang JY, Xu J. Gastric cancer and gene copy number variation: emerging cancer drivers for targeted therapy. Oncogene. 2015.

25. Wang H, Liang L, Fang JY, Xu J. Somatic gene copy number alterations in colorectal cancer: new quest for cancer drivers and biomarkers. Oncogene. 2015.

26. Hermeking $H$. MicroRNAs in the p53 network: micromanagement of tumour suppression. Nature Reviews Cancer. 2012; 12:613-626.

27. Hu Y, Wang J, Qian J, Kong X, Tang J, Wang Y, Chen H, Hong J, Zou W, Chen Y, Xu J, Fang JY. Long noncoding RNA GAPLINC regulates CD44-dependent cell invasiveness and associates with poor prognosis of gastric cancer. Cancer research. 2014; 74:6890-6902.

28. Ferreira-Teixeira M, Parada B, Rodrigues-Santos P, Alves V, Ramalho JS, Caramelo F, Sousa V, Reis F, Gomes CM. Functional and molecular characterization of cancer stemlike cells in bladder cancer: a potential signature for muscleinvasive tumors. Oncotarget. 2015; 6:36185-201. doi: 10.18632/oncotarget.5517.

29. Ahmed KA, Chinnaiyan P, Fulp WJ, Eschrich S, TorresRoca JF, Caudell JJ. . The radiosensitivity index predicts for overall survival in glioblastoma. Oncotarget. 2015; 6:34414-22. doi: 10.18632/oncotarget.5437.

30. Guo L, Lobenhofer EK, Wang C, Shippy R, Harris SC, Zhang L, Mei N, Chen T, Herman D, Goodsaid FM, Hurban P, Phillips KL, Xu J, et al. Rat toxicogenomic study reveals analytical consistency across microarray platforms. Nat Biotechnol. 2006; 24:1162-1169.

31. The MicroArray Quality Control (MAQC) project shows inter- and intraplatform reproducibility of gene expression measurements. Nat Biotechnol. 2006; 24:1151-1161.

32. Shi L, Jones WD, Jensen RV, Harris SC, Perkins RG, Goodsaid FM, Guo L, Croner LJ, Boysen C, Fang H, Qian F, Amur S, Bao W, et al. The balance of reproducibility, sensitivity, and specificity of lists of differentially expressed genes in microarray studies. BMC Bioinformatics. 2008; 9:S10.

33. Landi MT, Zhao Y, Rotunno M, Koshiol J, Liu H, Bergen AW, Rubagotti M, Goldstein AM, Linnoila I, Marincola FM. MicroRNA expression differentiates histology and predicts survival of lung cancer. Clinical Cancer Research. 2010; $16: 430-441$.

34. Jain N, Thatte J, Braciale T, Ley K, O’Connell M, Lee JK. Local-pooled-error test for identifying differentially expressed genes with a small number of replicated microarrays. Bioinformatics. 2003; 19:1945-1951.
35. Zhao H, Shen J, Medico L, Wang D, Ambrosone CB, Liu S. A pilot study of circulating miRNAs as potential biomarkers of early stage breast cancer. PloS one. 2010; 5:e13735.

36. Vickers KC, Palmisano BT, Shoucri BM, Shamburek RD, Remaley AT. MicroRNAs are transported in plasma and delivered to recipient cells by high-density lipoproteins. Nature cell biology. 2011; 13:423-433.

37. Hunten S, Kaller M, Drepper F, Oeljeklaus S, Bonfert T, Erhard F, Dueck A, Eichner N, Friedel CC, Meister G, Zimmer R, Warscheid B, Hermeking H. p53-Regulated Networks of Protein, mRNA, miRNA, and lncRNA Expression Revealed by Integrated Pulsed Stable Isotope Labeling With Amino Acids in Cell Culture (pSILAC) and Next Generation Sequencing (NGS) Analyses. Molecular \& cellular proteomics : MCP. 2015; 14:2609-2629.

38. Cancer Genome Atlas Research N. Comprehensive molecular characterization of gastric adenocarcinoma. Nature. 2014; 513:202-209.

39. Chen H, Xu J, Hong J, Tang R, Zhang X, Fang JY. Long noncoding RNA profiles identify five distinct molecular subtypes of colorectal cancer with clinical relevance. Molecular oncology. 2014; 8:1393-1403.

40. Buffa FM, Camps C, Winchester L, Snell CE, Gee HE, Sheldon H, Taylor M, Harris AL, Ragoussis J. microRNAassociated progression pathways and potential therapeutic targets identified by integrated mRNA and microRNA expression profiling in breast cancer. Cancer research. 2011; $71: 5635-5645$.

41. Vlachos IS, Zagganas K, Paraskevopoulou MD, Georgakilas G, Karagkouni D, Vergoulis T, Dalamagas T, Hatzigeorgiou AG. DIANA-miRPath v3.0: deciphering microRNA function with experimental support. Nucleic acids research. 2015; 43:W460-466.

42. Halder G, Camargo FD. The hippo tumor suppressor network: from organ size control to stem cells and cancer. Cancer research. 2013; 73:6389-6392.

43. Saito A, Nagase T. Hippo and TGF-beta interplay in the lung field. American journal of physiology Lung cellular and molecular physiology. Am J Physiol Lung Cell Mol Physiol. 2015; 309:L756-67.

44. Mullen AC. Hippo tips the TGF-beta scale in favor of pluripotency. Cell stem cell. 2014; 14:6-8.

45. Rihani A, Van Goethem A, Ongenaert M, De Brouwer S, Volders PJ, Agarwal S, De Preter K, Mestdagh P, Shohet J, Speleman F, Vandesompele J, Van Maerken T. Genome wide expression profiling of p53 regulated miRNAs in neuroblastoma. Scientific reports. 2015; 5:9027.

46. Krell J, Frampton AE, Colombo T, Gall TM, De Giorgio A, Harding V, Stebbing J, Castellano L. The p53 miRNA interactome and its potential role in the cancer clinic. Epigenomics. 2013; 5:417-428.

47. Sasaki A, Udaka Y, Tsunoda Y, Yamamoto G, Tsuji M, Oyamada H, Oguchi K, Mizutani T. Analysis of p53 and 
miRNA expression after irradiation of glioblastoma cell lines. Anticancer research. 2012; 32:4709-4713.

48. Garritano S, Inga A, Gemignani F, Landi S. More targets, more pathways and more clues for mutant p53. Oncogenesis. 2013; 2:e54.

49. Pan D. YAPing Hippo Forecasts a New Target for Lung Cancer Prevention and Treatment. Journal of clinical oncology. 2015; 33:2311-2313.

50. Song S, Ajani JA, Honjo S, Maru DM, Chen Q, Scott AW, Heallen TR, Xiao L, Hofstetter WL, Weston B, Lee JH, Wadhwa R, Sudo K, et al. Hippo coactivator YAP1 upregulates SOX9 and endows esophageal cancer cells with stem-like properties. Cancer research. 2014; 74: 4170-4182.

51. Kim MH, Seo HJ, Joung JG, Kim JH. Comprehensive evaluation of matrix factorization methods for the analysis of DNA microarray gene expression data. BMC bioinformatics. 2011; 12:S8.

52. Chu VT, Gottardo R, Raftery AE, Bumgarner RE, Yeung KY. $\mathrm{MeV}+\mathrm{R}$ : using $\mathrm{MeV}$ as a graphical user interface for Bioconductor applications in microarray analysis. Genome biology. 2008; 9:R118. 